\title{
Quark mass dependence of Spin-Orbit force in parity-odd NN system from 2+1 flavor QCD
}

\section{K. Murano for HAL-QCD Collaboration*}

Yukawa Institute for Theoretical Physics, Kyoto University, Kitashirakawa Oiwakecho,

Sakyo-ku, Kyoto 606-8502, Japan

E-mail: murano@yukawa.kyoto-u.ac.jp

We report our recent study of spin-orbit force between two nucleons in the parity-odd sector from lattice QCD. To examine the quark mass dependence of spin-orbit force, we construct it from Nambu-Bethe-Salpeter wave functions in ${ }^{3} P_{0},{ }^{3} P_{1}$ and ${ }^{3} P_{2}-{ }^{3} F_{2}$ channels calculated from lattice QCD in the quark mass region $m_{\pi}=702-411 \mathrm{MeV}$. The calculation is performed on Blue Gene/Q at KEK by using $N_{f}=2+1$ PACS-CS gauge configuration generated by $\mathscr{O}(a)$ improved Wilson quark action with RG improved (Iwasaki) gauge action. We find that the potentials tend to become stronger as the quark mass decreases.

31st International Symposium on Lattice Field Theory - LATTICE 2013

July 29 - August 3, 2013

Mainz, Germany

\footnotetext{
${ }^{*}$ Speaker.
} 


\section{Introduction}

The study of the nuclear force is a first step toward the understanding of the atomic nuclei beyond the single nucleon. The nuclear force plays a key role in describing various properties of atomic nuclei and neutron stars [1, 2]. Recently, a method to extract hadronic interactions from lattice QCD has been proposed, where energy-independent non-local potentials are defined from the Schrödinger equation by using the equal-time Nambu-Bethe-Salpeter (NBS) wave functions [3, 4]. The method gives the potentials which are faithful to the scattering phase shift. This is supported by the asymptotic long distance behavior of the equal-time NBS wave functions [4]. It has been successfully applied to the central and tensor forces in the parity-even NN system [3, 4, 5]. It has been extended and applied to various other systems such as hyperon-nucleon (YN), hyperonhyperon (YY), meson-baryon, and three-nucleons (NNN) [6]. Once these potentials are obtained, they can be used to study various physical observables such as bound states and scattering phase shift, by solving the Schrödinger equation. (See Ref.[7] for a comparison between the Lüscher's method and the potential method for the $\pi \pi$ scattering phase shifts, where a good agreement is obtained.)

The potentials are classified order by order in the derivative expansion of the non-local potentials. At the leading order (LO), we have the spin-singlet central potential $V_{\mathrm{C} ; \mathrm{S}=0}^{( \pm)}(r)$, the spin-triplet central potential $V_{\mathrm{C} ; \mathrm{S}=1}^{( \pm)}(r)$ and the tensor potential $V_{\mathrm{T}}^{( \pm)}(r)$, where the super-index “ $( \pm)$ " indicates the parity of the two-nucleon system. At the next-to-leading order (NLO), there appear the spinorbit potentials $V_{\mathrm{LS}}^{( \pm)}(r)$. Up to the NLO, there are these 8 independent local potentials [4]. So far our studies have been mainly concentrated on the central and the tensor potentials in the parityeven sectors which can be obtained from the NBS wave functions in $S$ and $D$ waves [3, 4, 5]. For complete determination of NN potentials, we need also to determine the central and the tensor potentials in the parity-odd sector at LO as well as the spin-orbit (LS) potentials in both parity sectors at NLO. Especially, the spin-orbit potential is known to be important in the NN system not only to reproduce the experimental phase shift in spin-triplet $P$ wave channels, but also to induce the $P$-wave superfluidity in the stellar environment such as the neutron star interiors [8, 9, 10].

As our recent progress, we have extended our method to the central, the tensor and the spinorbit potentials in the parity-odd sector, and have presented the first result of these potentials [11]. The calculation was performed by using the 2 flavor gauge configuration generated by CP-PACS collaboration at $m_{\pi}=1133 \mathrm{MeV}$ [12], where we found that, while the qualitative behavior of resultant potentials are consistent with phenomenological ones, these potentials are still weak, which is considered to be caused by the heavy quark mass employed in our simulations. The main purpose of this paper is to examine the quark mass dependence of the central, the tensor and the spin-orbit potentials in parity-odd $\mathrm{NN}$ system, by using the $2+1$ flavor gauge configurations generated by PACS-CS collaboration at $m_{\pi}=702,570$ and $411 \mathrm{MeV}$ [13].

\section{Definition of the potential}

To construct the NN potential, we consider the equal-time Nambu-Bethe-Salpeter (NBS) wave function in the center of mass (CM) frame, defined by

$$
\phi_{\alpha, \beta}(\mathbf{r} ; \mathbf{k}) \equiv\left\langle 0\left|p_{\alpha}(\mathbf{x}) n_{\beta}(\mathbf{y})\right| p(+\mathbf{k}) n(-\mathbf{k})\right\rangle, \quad(\mathbf{r} \equiv \mathbf{x}-\mathbf{y})
$$


where $p_{\alpha}(\mathbf{x})$ and $n_{\beta}(\mathbf{y})$ denote local composite nucleon operators with spinor indices $\alpha, \beta$, and $\mathbf{k}$ denotes the asymptotic momentum of the two-nucleon state. We define NN potentials from the NBS wave function below the inelastic threshold $\left(E<E_{\text {th }} \equiv 2 m_{N}+m_{\pi}\right)$ by using the Schrödinger equation 4, 6]

$$
\left(E_{\mathbf{k}}+\frac{\nabla^{2}}{m_{N}}\right) \phi(\mathbf{r} ; \mathbf{k})=\int d^{3} r\left(\mathbb{P}^{(+)} U^{(+)}\left(\mathbf{r}, \mathbf{r}^{\prime}\right)+\mathbb{P}^{(-)} U^{(-)}\left(\mathbf{r}, \mathbf{r}^{\prime}\right)\right) \phi\left(\mathbf{r}^{\prime} ; \mathbf{k}\right),
$$

where $E_{\mathrm{k}} \equiv \frac{\mathbf{k}^{2}}{m_{N}}$ denotes the non-relativistic energy. $\mathbb{P}^{(+)}$and $\mathbb{P}^{(-)}$denote projection operators for parity-even and parity-odd sectors, respectively. $U^{(+)}$and $U^{(-)}$denote the non-local potentials for parity-even and parity-odd sectors, to which we apply the derivative expansion as

$$
\begin{aligned}
& U^{( \pm)}\left(\mathbf{r}, \mathbf{r}^{\prime}\right)=V^{( \pm)}(\mathbf{r}, \nabla) \delta\left(\mathbf{r}-\mathbf{r}^{\prime}\right) \\
& V^{( \pm)}(\mathbf{r}, \nabla)=V_{\mathrm{C} ; \mathrm{S}=0}^{( \pm)}(r) \mathbb{P}^{(S=0)}+V_{\mathrm{C} ; \mathrm{S}=1}^{( \pm)}(r) \mathbb{P}^{(S=1)}+V_{T}^{( \pm)}(r) S_{12}+V_{L S}^{( \pm)}(r) \mathbf{L} \cdot \mathbf{S}+(\mathrm{NNLO}),
\end{aligned}
$$

where $\mathbb{P}^{(S=0)} \equiv\left(1-\sigma_{1} \cdot \sigma_{2}\right) / 4$ and $\mathbb{P}^{(S=1)} \equiv\left(3+\sigma_{1} \cdot \sigma_{2}\right) / 4$ denote the projection operators onto the total spin singlet and triplet sectors, respectively. $S_{12} \equiv 3\left(\mathbf{r} \cdot \sigma_{1}\right)\left(\mathbf{r} \cdot \sigma_{2}\right) / \mathbf{r}^{2}-\sigma_{1} \cdot \sigma_{2}$ is referred to as the tensor operator. $\mathbf{L} \equiv i \mathbf{r} \times \nabla$ and $\mathbf{S} \equiv\left(\sigma_{1}+\sigma_{2}\right) / 2$ denote the orbital angular momentum operator and the total spin operator, respectively. $V_{\mathrm{C} ; \mathrm{S}=0}^{( \pm}, V_{\mathrm{C} ; \mathrm{S}=1}^{( \pm)}, V_{\mathrm{T}}^{( \pm)}$and $V_{\mathrm{LS}}^{( \pm)}$are referred to as the spin-singlet central, the spin-triplet central, the tensor, and the spin-orbit forces, respectively. While $V_{\mathrm{C} ; \mathrm{S}=0}^{( \pm)}, V_{\mathrm{C} ; \mathrm{S}=1}^{( \pm)}$and $V_{\mathrm{T}}^{( \pm)}$are of leading $\operatorname{order}(\mathrm{LO})$ in the derivative expansion of the nonlocal potential, $V_{\mathrm{LS}}^{( \pm)}$appears at next-to-leading order(NLO). Once the above NBS wave functions are calculated in lattice QCD simulations, these potentials can be extracted by solving Eq.2.2.

\subsection{Spin-triplet potentials in parity-odd sector including spin-orbit force}

We restrict ourselves to the spin-triplet and parity-odd sector. The Schrödinger equation Eq.(2.2) reads

$$
\left(E+\frac{\nabla^{\mathbf{2}}}{m_{N}}\right) \phi(\mathbf{r})=\left[V_{\mathrm{C} ; \mathrm{S}=1}^{(-)}(r)+V_{\mathrm{T}}^{(-)}(r) S_{12}+V_{\mathrm{LS}}^{(-)}(r) \mathbf{L} \cdot \mathbf{S}\right] \phi(\mathbf{r}) .
$$

In order to determine $V_{\mathrm{C} ; \mathrm{S}=1}^{(-)}, V_{\mathrm{T}}^{(-)}$and $V_{\mathrm{LS}}^{(-)}$, we need three independent NBS wave functions $\phi_{i}(\mathbf{r})$ with $i=1,2,3$, for which we take NBS wave functions in ${ }^{3} P_{0}\left(J^{P}=0^{-}\right),{ }^{3} P_{1}\left(J^{P}=1^{-}\right)$and ${ }^{3} P_{2}-{ }^{3} F_{2}\left(J^{P}=2^{-}\right)$channels. $V_{\mathrm{C} ; \mathrm{S}=1}^{(-)}, V_{\mathrm{T}}^{(-)}$and $V_{\mathrm{LS}}^{(-)}$are obtained as solutions to Eq. (2.4) as

$$
\left(\begin{array}{c}
V_{\mathrm{C} ; \mathrm{S}=1}^{(-)}(r) \\
V_{\mathrm{T}}^{(-)}(r) \\
V_{\mathrm{LS}}^{(-)}(r)
\end{array}\right)=M(\vec{r})^{-1}\left(\begin{array}{c}
\left(\nabla^{2} / m_{N}+E_{1}\right) \phi_{1}(\mathbf{r}) \\
\left(\nabla^{2} / m_{N}+E_{2}\right) \phi_{2}(\mathbf{r}) \\
\left(\nabla^{2} / m_{N}+E_{3}\right) \phi_{3}(\mathbf{r})
\end{array}\right)
$$

where $E_{i}$ denotes the non-relativistic energy associated with the NBS wave functions $\phi_{i}(\mathbf{r})$, and $M(\vec{r})$ is a $3 \times 3$ matrix defined by

$$
M(\vec{r}) \equiv\left(\begin{array}{lll}
\phi_{1}(\mathbf{r}) & S_{12} \phi_{1}(\mathbf{r}) & \mathbf{L} \cdot \mathbf{S} \phi_{1}(\mathbf{r}) \\
\phi_{2}(\mathbf{r}) & S_{12} \phi_{2}(\mathbf{r}) & \mathbf{L} \cdot \mathbf{S} \phi_{2}(\mathbf{r}) \\
\phi_{3}(\mathbf{r}) & S_{12} \phi_{3}(\mathbf{r}) & \mathbf{L} \cdot \mathbf{S} \phi_{3}(\mathbf{r})
\end{array}\right) .
$$




\section{Construction of NBS wave functions by lattice QCD}

The equal-time NBS wave functions are obtained from 4-point nucleon correlation functions on the lattice in the large $t$ region as

$$
\begin{aligned}
G\left(\mathbf{x}-\mathbf{y}, t-t_{0} ; \mathscr{J}^{J, S=1}\right) & \equiv \frac{1}{L^{3}} \sum_{\mathbf{r}}\left\langle 0\left|T\left[p(\mathbf{x}+\mathbf{r}, t) n(\mathbf{y}+\mathbf{r}, t) \mathscr{J}^{J, S=1}\left(t_{0}\right)\right]\right| 0\right\rangle \\
& \simeq \phi_{0}^{J, S=1}(\mathbf{x}-\mathbf{y}) a_{0} e^{-E_{0}\left(t-t_{0}\right)}, \quad t-t_{0} \gg 1,
\end{aligned}
$$

where the summation over $\mathbf{r}$ in the first line is performed to select the two-nucleon system with vanishing total spatial momentum. $\mathscr{J}^{J, S=1}$ denotes a two-nucleon source operator for the total angular momentum $J$ in the spin-triplet parity-odd sector. For nucleon operators $p(x)$ and $n(y)$, we employ the following local composite operators

$$
p(x) \equiv \varepsilon_{a b c}\left(u_{a}^{T}(x) C \gamma_{5} d_{b}(x)\right) u_{c}(x), \quad n(x) \equiv \varepsilon_{a b c}\left(u_{a}^{T}(x) C \gamma_{5} d_{b}(x)\right) d_{c}(x),
$$

where $a, b$ and $c$ denote color indices. $\phi_{0}^{J, S=1}(\mathbf{r})$ and $E_{0}$ denotes the NBS wave function and the energy of the ground state in the total angular momentum $J$ in the spin-triplet parity-odd sector, respectively. The coefficient $a_{0} \equiv\left\langle p\left(+\mathbf{k}_{0}\right) n\left(-\mathbf{k}_{0}\right)\left|\mathscr{J}^{J, S=1}(0)\right| 0\right\rangle$ is the overlap factor between states created by the source and the ground state in this system with the asymptotic momentum $\mathbf{k}_{0}$.

For the two-nucleon source operator $\mathscr{J}^{J, S=1}$, we take two-nucleon momentum wall source operator defined by

$$
\mathscr{J}_{\alpha \beta}(f) \equiv \bar{P}_{\alpha}(f) \bar{N}_{\beta}\left(f^{*}\right),
$$

where

$$
\begin{aligned}
& \bar{P}_{\alpha}(f) \equiv \sum_{\mathbf{x}_{1}, \mathbf{x}_{2}} \varepsilon_{a b c}\left(\bar{u}_{a}\left(\mathbf{x}_{1}\right) C \gamma_{5} \bar{d}_{b}^{T}\left(\mathbf{x}_{2}\right)\right) \sum_{\mathbf{x}_{3}} \bar{u}_{c, \alpha}\left(\mathbf{x}_{3}\right) f\left(\mathbf{x}_{3}\right) \\
& \bar{N}_{\beta}(f) \equiv \sum_{\mathbf{x}_{1}, \mathbf{x}_{2}} \varepsilon_{a b c}\left(\bar{u}_{a}\left(\mathbf{x}_{1}\right) C \gamma_{5} \bar{d}_{b}^{T}\left(\mathbf{x}_{2}\right)\right) \sum_{\mathbf{x}_{3}} \bar{d}_{c, \beta}\left(\mathbf{x}_{3}\right) f\left(\mathbf{x}_{3}\right)
\end{aligned}
$$

with $f$ being one of the following source functions, each of which corresponds to a plane wave parallel or anti-parallel to one of the spatial coordinate axes as

$$
\begin{array}{lll}
f^{(0)}(\mathbf{r}) \equiv \exp (-2 \pi i x / L), & f^{(1)}(\mathbf{r}) \equiv \exp (-2 \pi i y / L), & f^{(2)}(\mathbf{r}) \equiv \exp (-2 \pi i z / L), \\
f^{(3)}(\mathbf{r}) \equiv \exp (+2 \pi i x / L), & f^{(4)}(\mathbf{r}) \equiv \exp (+2 \pi i y / L), & f^{(5)}(\mathbf{r}) \equiv \exp (+2 \pi i z / L) .
\end{array}
$$

Note that an element $g$ of the cubic group $O$ with 24 elements acts on these plane waves as

$$
f^{(i)} \mapsto \sum_{j} U_{i j}(g) f^{(j)}
$$

where $U(g)$ is a $6 \times 6$ permutation matrix, which servers as the representation matrix of $g \in O$. By a cubic group analysis, the orbital part of this momentum wall source is decomposed into $A_{1}^{+} \oplus E^{+} \oplus$ $T_{1}^{-}$. Therefore, for the parity-odd sector, we can access $J^{P}=\left(L=T_{1}^{-}\right) \otimes\left(S=A_{1}\right)=T_{1}^{-}\left(\simeq 1^{-}\right)$for the spin-singlet sector and $J^{P}=\left(L=T_{1}^{-}\right) \otimes\left(S=T_{1}\right)=A_{1}^{-} \oplus E^{-} \oplus T_{1}^{-} \oplus T_{2}^{-}\left(\simeq 0^{-} \oplus 2^{-} \oplus 1^{-} \oplus 2^{-}\right)$ for the spin-triplet sector. 
The momentum wall source with a definite total angular momentum is now constructed as

$$
\mathscr{J}_{\alpha \beta}^{J}\left(f^{(i)}\right) \equiv \frac{d^{(J)}}{24} \sum_{g \in O} \chi^{(J)}\left(g^{-1}\right) U_{i j}(g) \mathscr{J}_{\alpha^{\prime} \beta^{\prime}}\left(f^{(j)}\right) S_{\alpha^{\prime} \alpha}^{-1}\left(g^{-1}\right) S_{\beta^{\prime} \beta}^{-1}\left(g^{-1}\right)
$$

where $d^{(J)}$ and $\chi^{(J)}(g)$ denote the dimension and the character for the irreducible representation $J$ of the cubic group, respectively. Hereafter the Dirac indices $\alpha, \beta$ are restricted to upper components (in the Dirac representation). The total spin $S$ is projected by the spin projection operator $\mathbb{P}^{(S)}$ as

$$
\mathscr{J}_{\alpha \beta}^{J, S}\left(f^{(i)}\right) \equiv \mathbb{P}_{\alpha \beta, \gamma \delta}^{(S)} \mathscr{J}_{\gamma \delta}^{J}\left(f^{(i)}\right)
$$

Finally, the parity projection is performed by

$$
\mathbb{P}^{( \pm)} \mathscr{J}_{\alpha \beta}\left(f^{(i)}\right) \equiv \frac{1}{2}\left(\mathscr{J}_{\alpha \beta}\left(f^{(i)}\right) \pm \mathscr{J}_{\alpha \beta}\left(f^{(i) *}\right)\right) .
$$

Note that $f^{(i) *}$ is the plane wave with the opposite momentum of $f^{(i)}$. (For detail of the construction of two-nucleon momentum wall source operator, see Ref.[11])

\section{Numerical results}

Our calculation is performed by using $N_{f}=2+1$ full QCD gauge configurations generated by PACS-CS collaboration on a $32^{3} \times 64$ lattice [13], which employs the RG improved action (Iwasaki action) at $\beta=1.90$ leading to the lattice spacing $a^{-1}=2.176(31) \mathrm{GeV}(a=0.0907(13) \mathrm{fm})$ and the lattice extension $L \simeq 2.9 \mathrm{fm}$. As the quark action, it employs the $\mathscr{O}(a)$ improved Wilson quark action (clover action) with $C_{\mathrm{SW}}=1.715$ at $\kappa_{u d}=0.13700,0.13727$ and 0.13754 and $\kappa_{s}=0.13640$. These $\kappa_{u d}$ correspond to the pion mass $m_{\pi}=702(1)(10), 570(2)(8)$ and $411.3(2)(6) \mathrm{MeV}$ and the nucleon mass $m_{N}=1538(5)(23), 1411(12)(20)$ and 1215(12)(17) MeV, respectively. The first errors are statistical and the second ones are the systematic errors coming from ambiguity of the lattice scale. The 4-point nucleon correlation functions Eq. 3.10 are calculated with the periodic and Dirichlet boundary conditions along the spatial and the temporal directions, respectively. To improve the statistics, we use 4 source points by temporally shifting the gauge configurations. The charge conjugation and time reversal symmetries are used to double the number of statistical data.

We calculate the NBS wave functions with $J^{P}=A_{1}^{-}, T_{1}^{-}$and $E^{-}$, whose dominant components correspond to ${ }^{3} P_{0},{ }^{3} P_{1}$ and ${ }^{3} P_{2}-{ }^{3} F_{2}$, respectively. In order to extract the potentials, we solve Eq. [2.4) by using the time-dependent method[5] which can efficiently extract the potentials without requiring the ground state saturation of the 4-point nucleon correlation functions Eq. 3.1].

Preliminary results of the central, the tensor and the spin-orbit potentials at $t-t_{0}=8,9,10$ for $m_{\pi}=702 \mathrm{MeV}$ are presented in Fig.1] We see similar behaviors observed in Ref.[11] such as (1) the central potential has a repulsive core at short distance, (2) the tensor potential is weak and positive, and (3) the spin-orbit potential is negative and strong. However, we observe that the convergence of long distance part of the central potential is very slow, which may be caused by the NNLO terms in the derivative expansion of the non-local potential or the inelastic contribution in the 4-point nucleon correlation function Eq. 3.1. Similar tendency is seen for the case of other quark masses. To achieve the time slice saturation, we need to use the NBS wave functions at 

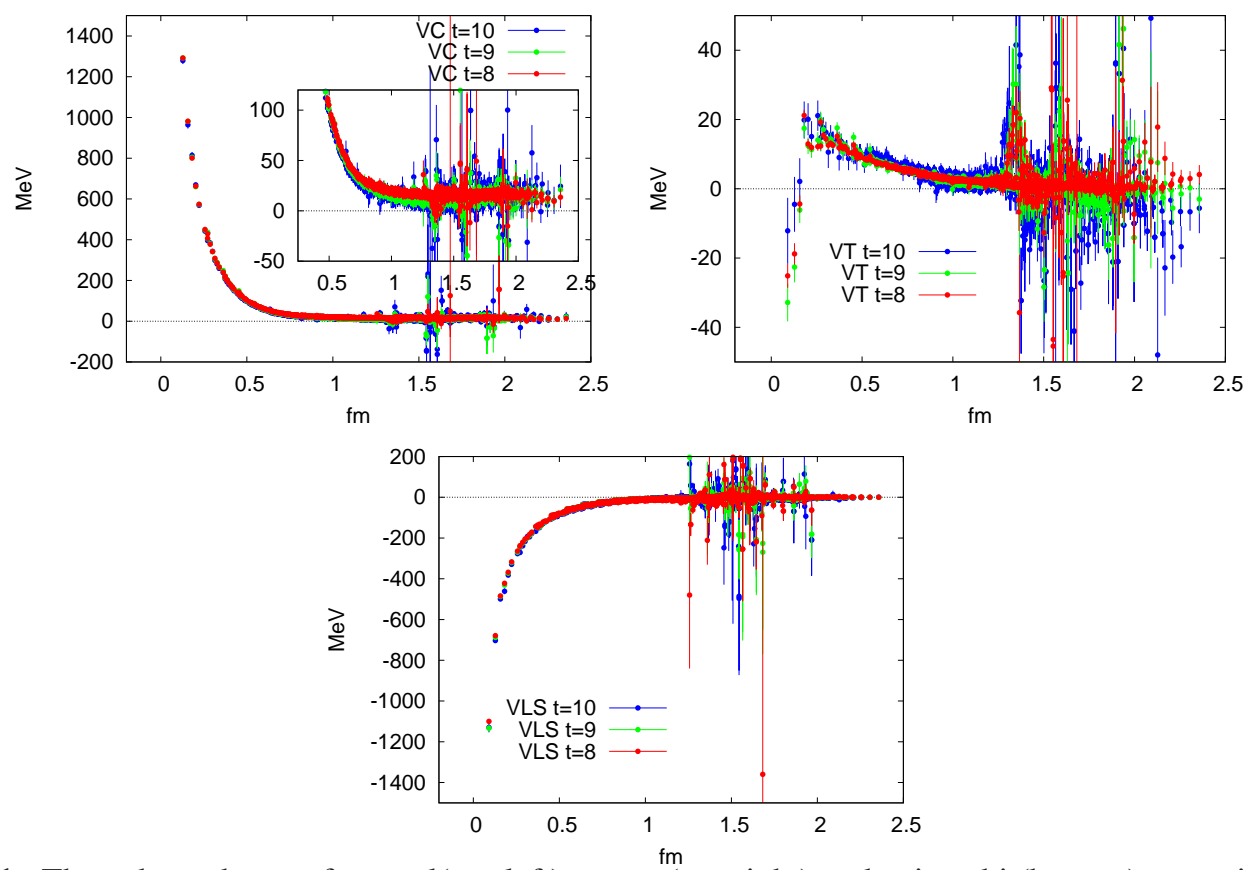

Figure 1: The $t$-dependence of central(top left), tensor(top right) and spin-orbit(bottom) potentials in the spin-triplet and parity odd sector for $t-t_{0}=8$ (red), 9(green) and 10(blue) for pion masses $702 \mathrm{MeV}$.

somewhat larger $t$. Preliminary results on the quark mass dependence of the central, the tensor, and the spin-orbit potentials at $t-t_{0}=9$ are shown in Fig 2. Red, blue and black data correspond to the pion mass $m_{\pi}=702,570$ and $411 \mathrm{MeV}$, respectively. We find the tendency that these potentials become stronger as the quark mass decreases. In order to discuss the behaviors of the P-wave phase shifts, it is necessary to achieve the time slice saturation of the central potential. For this, it is important to increase the statistics.

\section{Summary}

As a continuation of our previous studies of the central, the tensor and the spin-orbit potential in the parity-odd sector, we have examined a quark mass dependence of these potentials by using $2+1$ flavor gauge configurations which are generated by PACS-CS collaboration at $m_{\pi}=702,570$ and $411 \mathrm{MeV}$. Although the time slice saturation is not achieved yet, we have observed the tendency that these potentials become stronger as the quark mass decreases.

The lattice QCD calculation has been done on Blue Gene/Q at KEK under the support of the Large Scale Simulation Program No.12/13-19(FY2013) and No.12-11(FY2012) of High Energy Accelerator Research Organization (KEK). We are grateful for authors and maintainers of CPS++[14], a modified version of which is used for simulations done in this report. We thank PACS-CS collaboration [13] and ILDG/JLDG [15] for 2+1 flavor QCD gauge configurations. This research is supported in part by MEXT Grant-in-Aid for Scientific Research (No.25287046), for Scientific Research on Innovative Areas (No.2004: 20105001, 20105003) and SPIRE (Strategic Program for Innovative REsearch). 


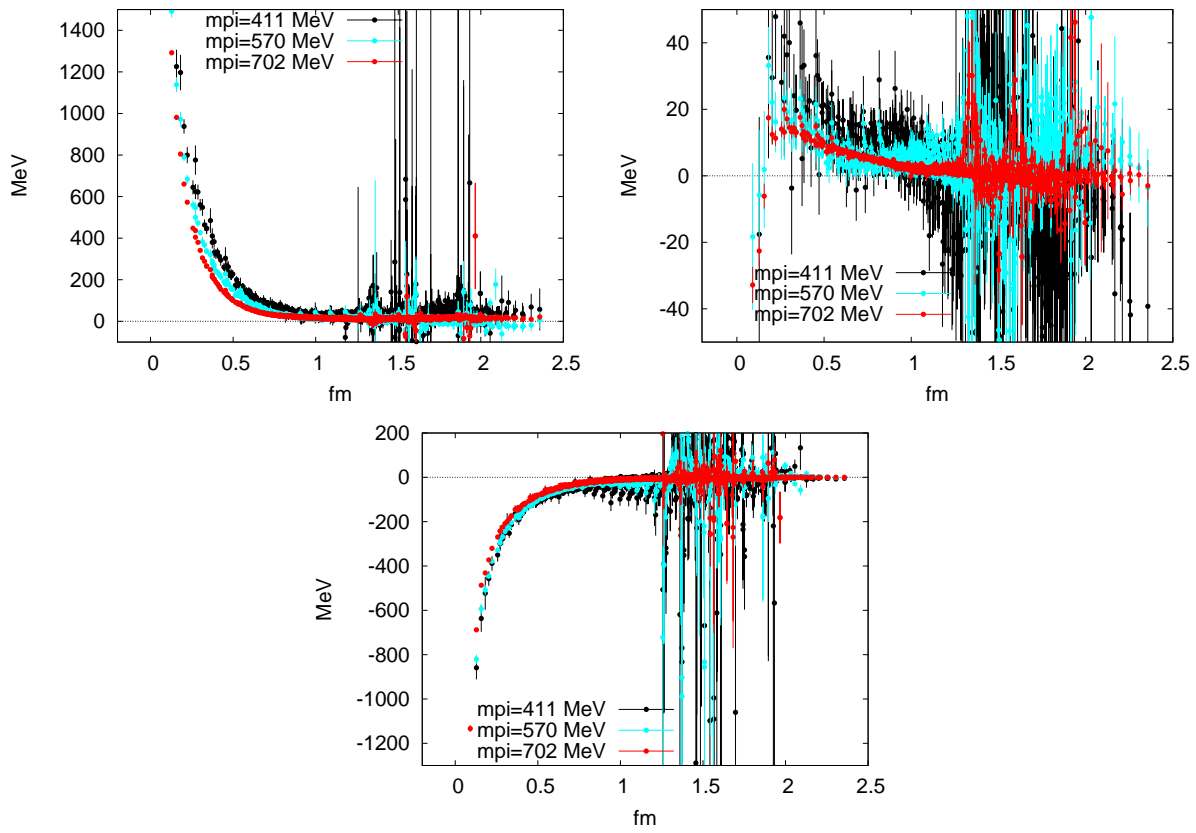

Figure 2: The quark mass dependence of the central potential (left), tensor potential (right) and spin-orbit potential (bottom) in the spin-triplet and parity-odd sector. Red, blue and black points corresponding to $m_{\pi}=702,570$ and $411 \mathrm{MeV}$, respectively.

\section{References}

[1] E. Epelbaum, H. -W. Hammer, U. -G. Meissner, Rev. Mod. Phys. 81 (2009) 1773-1825.

[2] R. Machleidt and D. R. Entem, Phys. Rept. 503 (2011) 1.

[3] N. Ishii, S. Aoki and T. Hatsuda, Phys. Rev. Lett. 99 (2007) 022001.

[4] S. Aoki, T. Hatsuda and N. Ishii, Prog. Theor. Phys. 123 (2010) 89.

[5] N. Ishii et al. [HAL QCD Collaboration], Phys. Lett. B 712 (2012) 437.

[6] Reviewed in, S. Aoki et al. [HAL QCD Collaboration], PTEP 2012 (2012) 01A105.

[7] T. Kurth, N. Ishii, T. Doi, S. Aoki and T. Hatsuda, arXiv:1305.4462 [hep-lat].

[8] R. Tamagaki, Prog. Theor. Phys. 44, 905 (1970). T. Takatsuka and R. Tamagaki, 46 (1971) 114. T. Takatsuka, 47, 1062 (1972), ibid. Prog. Theor. Phys. 48, 1517 (1972).

[9] M. Hoffberg, A.E. Glassgold, R.W. Richardson, M. Rudermann, Phys. Rev. Lett. 24 (1970) 775.

[10] M. Baldo, O. Elgaroey, L. Engvik, M. Hjorth-Jensen and H. J. Schulze, Phys. Rev. C 58 (1998) 1921.

[11] K. Murano, N. Ishii, S. Aoki, T. Doi, T. Hatsuda, Y. Ikeda, T. Inoue and H. Nemura et al., arXiv:1305.2293 [hep-lat].

[12] A. Ali Khan et al. [CP-PACS Collaboration], Phys. Rev. D 65 (2002) 054505 [Erratum-ibid. D 67 (2003) 059901].

[13] S. Aoki et al. [PACS-CS Collaboration], Phys. Rev. D 79, 034503 (2009).

[14] Columbia Physics System (CPS), http://qcdoc.phys.columbia.edu/cps.html

[15] http://www.lqcd.org/ildg; http://www.jldg.org/ 